О ВРЕМЕННЫХ ЗАДЕРЖКАХ

МЕЖДУ СУБ-ТЕРАГЕЦОВЫМ И МЯГКИМ РЕНТГЕНОВСКИМ ИЗЛУЧЕНИЕМ СОЛНЕЧНЫХ ВСПЫШЕК

\author{
Смирнова В.В. ${ }^{1}$, Цап Ю.Т. ${ }^{1}$, Моторина Г.Г. ${ }^{2,3}$, Моргачев А.С. ${ }^{4}$ \\ ${ }^{1}$ Крымская астрофизическая обсерватория РАН, $n$. Научный, Крым, Россия \\ ${ }^{2}$ Главная (Пулковская) астрономическая обсерватория РАН, Санкт-Петербург, Россия \\ ${ }^{3}$ Astronomical Institute ASCR, 25165 Ondrejov, Czech Republic \\ ${ }^{4}$ Нижегородский государственный университет им. Н.И. Лобачевского, \\ Нижний Новгород, Россия
}

\title{
ON THE TIME DELAYS BETWEEN SUB-TERAHERTZ AND SOFT X-RAY EMISSION FROM SOLAR FLARES
}

\author{
Smirnova V.V. ${ }^{1}$, Tsap Y.T. ${ }^{1}$, Motorina G.G. ${ }^{2,3}$, Morgachev A.S. ${ }^{4}$ \\ ${ }^{1}$ Crimean Astrophysical Observatory of RAS, Nauchny, Crimea, Russia \\ ${ }^{2}$ Pulkovo Observatory of RAS, St. Petersburg, Russia \\ ${ }^{3}$ Astronomical Institute ASCR, 25165 Ondrejov, Czech Republic \\ ${ }^{4}$ Radiophysical Research Institute of Lobachevsky University, Nizhny Novgorod, Russia
}

The time delays between peaks of sub-THz and soft X-ray emissions from solar flares have been considered. It has been shown that two types of sub-THz events exist depending on the time delays. This can be caused by different mechanisms of solar chromospheric heating during the flare energy release.

DOI: 10.31725/0552-5829-2020-269-272

\section{Введение}

Несмотря на то, что после обнаружения возрастающего с частотой потока излучения солнечных вспышек в суб-ТГц диапазоне $\left(10^{2}-10^{3}\right.$ Гц, 0.3-3.0 мм) прошло уже около 20 лет [1], тем не менее, вопрос о природе этого явления все еще остается открытым. За указанный период времени было предложено с десяток различных механизмов (моделей), способных объяснить положительный наклон спектра. Среди них: гиросинхротронный $[2,3]$, обратный комптоновский $[2]$, тепловой тормозной $[4,5]$, гибридный [6], черенковский [7] и плазменный механизмы [8]. Однако перечисленные модели встречаются с теми или иными трудностями $[6,8,9]$. В частности, черенковский механизм предполагает, что диэлектрическая проницаемость солнечной хромосферы должна быть больше 1, что представляется довольно сомнительным [9]. Для реализации гиросинхротронного механизма требуются магнитные поля в несколько тысяч гаусс [3], тогда как для плазменного необходима концентрация тепловых электронов, не менее $10^{14} \mathrm{~cm}^{-3}$, что может быть достигнуто в нижней атмосфере Солнца при условии эффективной ионизации атомов водорода.

Сравнительно недавно с целью выяснения природы суб-ТГц излучения солнечных вспышек с положительным наклоном спектра авторы рабо- 
ты [5] провели детальный статистический анализ известных вспышечных событий (15 событий). Было показано, что как наблюдаемые потоки, так и суб-секундные пульсации суб-ТГц излучения можно объяснить, исходя из предположения об определяющем вкладе теплового тормозного механизма, генерируемого на уровне хромосферы и переходной области в результате термализации ускоренных в короне электронов. Однако площадь источников излучения в работе [5] находилась по засвеченным изображениям. Другим важным недостатком этой работы является отсутствие анализа поведения временных профилей теплового и нетеплового излучения вспышек. Между тем следует ожидать, что в рамках рассматриваемой модели должен наблюдаться эффект Нойперта, который сводится в простейшем случае к наступлению максимума суб-ТГц излучения на несколько минут раньше мягкого рентгеновского (см. также [10]).

Цель настоящей работы - на основе известных вспышечных событий провести детальный анализ временных задержек между суб-ТГц и мягким рентгеновским излучением.

\section{Обработка данных наблюдений и ее результаты}

Исходя из работы [5], а также из других работах (например: [11-13]), мы выбрали ряд суб-ТГц событий с положительным спектром и сравнили временные профили радио- и мягкого рентгеновского излучения на предмет наличия временных задержек между ними. Для мягкого рентгеновского излучения (1-8, 0.5-4 А) использовались наблюдения космических аппаратов серии GOES [14], тогда как для суб-терагерцового - радиотелескопов SST, KOSMA, BEMRAK и РТ-7,5 МГТУ, на частотах 93, 140, 210, 230, 345 , 405 ГГц. Всего было выделено 11 событий (таблица 1). Временные задержки, превышающие 30 с, определялись между максимумами потоков субТГц и мягкого рентгеновского излучения (в противном случае, они во внимание не принимались).

Таблица 1.

\begin{tabular}{|l|l|l|l|l|l|}
\hline № & Тип & Дата и время & $\begin{array}{l}\text { GOЕS-класс } \\
\text { вспышки }\end{array}$ & $\begin{array}{l}\text { Задержки } \\
\text { (минуты) }\end{array}$ & Публикация \\
\hline 1 & I & $06.12 .2006(18: 44$ UT) & X6.5 & 3 & Kaufmann et al. (2009) \\
\hline 2 & I & $12.04 .2001(10: 17$ UT) & X2.0 & 9 & Luthi et al. (2004) \\
\hline 3 & I & $27.10 .2014(14: 22$ UT) & X2.0 & 6 & Fernandes et al. (2017) \\
\hline 4 & I & $2.11 .2003(17: 07$ UT) & X8.3 & 2,5 & Silva et al. (2007) \\
\hline 5 & I & $30.10 .2004(11: 40 ~ U T)$ & X1.2 & 2 & Cristiani et al. (2007) \\
\hline 6 & II & $05.07 .2012(11: 44$ UT) & M6.2 & - & Tsap et al. (2018) \\
\hline 7 & II & $04.07 .2012(09: 54$ UT) & M5.3 & - & Tsap et al. (2016) \\
\hline 8 & II & $02.04 .2017(07: 50$ UT) & M5.4 & - & Morgachev et al. (2018) \\
\hline 9 & II & $27.10 .2003(12: 27$ UT) & M6.7 & - & Trottet et al. (2011) \\
\hline 10 & II & $08.02 .2010(13: 30 ~ U T)$ & M2 & - & Kaufmann et al. (2011) \\
\hline 11 & I, II & $17.02 .2013(15: 40 ~ U T)$ & M1.9 & $*$ & Fernandes et al. (2017) \\
\hline
\end{tabular}

Символом (*) обозначена вспышка, попадающая под оба типа. 
Полученные результаты позволяют разделить суб-ТГц события на два типа:

I. вспышки, для которых появление максимума потока радиоизлучения опережает по времени появление максимума мягкого рентгеновского излучения от 2 до 9 минут;

II. вспышки, для которых максимумы потоков суб-ТГц и мягкого рентгеновского излучения совпадают с точностью до 30 с, по результатам кросс-корреляционного анализа.

Типичные примеры временных профилей вспышек типа I и типа II представлены на рис. 1. В первом случае (рис.1 а), задержка между профилями суб-ТГц и мягкого рентгеновского излучения составила 3 мин, во втором случае (рис. 1 b, с) максимумы совпадают, однако на рис.1 b видно, что в суб-ТГц диапазоне наблюдается небольшой импульс в начале вспышки.

Следует подчеркнуть, что к типу I относилось 5 событий класса $\mathrm{X}$, a ко второму - 5 вспышек класса М.

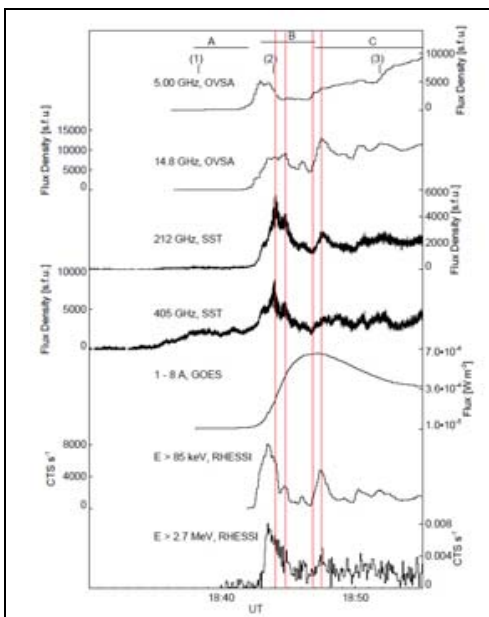

(a)

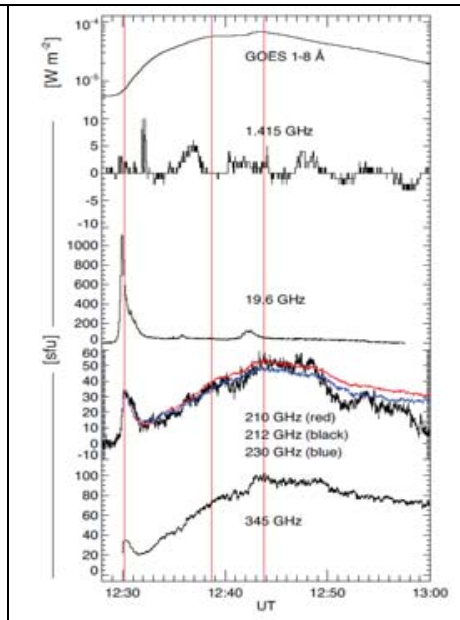

(b)

\begin{tabular}{|l|l|l} 
Kaufmann et al. (2009) [11] & Trottet et al. (2011) [12] & Morgachev et al. (2018) [13]
\end{tabular}

Рис. 1. Временные профили вспышек типа I (a) и типа II (b, c).

\section{Обсуждение результатов и выводы}

Полученные нами результаты, по меньшей мере, свидетельствуют о том, что для суб-ТГц событий с положительным наклоном спектра, предположение, сделанное в работе [5] о доминирующем механизме нагрева переходной области и хромосферы Солнца быстрыми электронами, не согласуется, по крайней мере, с некоторыми наблюдениями. В этом случае должны были бы доминировать вспышки типа I, что несколько противоречит полученным нами результатам. По нашему мнению, с учетом малого вклада горячей корональной плазмы в суб-ТГц излучение для вспышек рентгеновского класса М, это можно объяснить процессами теплового энерговыделения in situ на уровне хромосферы. Однако не следует также 
исключать определенный вклад и нетепловых процессов в нижней атмосфере Солнца. Данный вывод находится в хорошем согласии с результатами работы [15], согласно которой, по крайней мере, для некоторых событий в основаниях корональных петель наблюдается горячий $>10 \mathrm{MK}$ источник мягкого рентгеновского излучения, который никак не связан с последующей импульсной фазой вспышки.

Вместе с тем важную роль могут также играть процессы распространения тепловых потоков из корональной части вспышечной петли в основания петель [16]. Для того чтобы выяснить причину отсутствия временных задержек между мягким рентгеновским и суб-ТГц излучением требуются дополнительные исследования.

Работа выполнена при частичной поддержке Минобрнауки НИР № 0831-2019-0006 (Смирнова В.В., Цап Ю.Т.), и гранта РНФ 20-72-10158 (Моторина Г.Г.).

\section{Литература}

1. Trottet, G., Raulin, J.-P., Kaufmann, P., et al. // A\&A, 2002, 381, 694.

2. Kaufmann, P., Correia, E., Costa, J.E.R., Zodi Vaz, A.M. // A\&A, 1986, 157, 11.

3. Silva, A.V.R., Share, G.H., Murphy, R.J., et al. // Sol. Phys., 2007, 245, 311.

4. Tsap, Y.T., Smirnova, V.V., Morgachev, A.S., et al. // Adv. Space Res., 2016, 57, 1449.

5. Kontar, E.P., Motorina, G.G., and Jeffrey, N.L.S., et al. // A\&A, 2018, vol. 620, id A95.

6. Tsap Yu.T., Smirnova V.V., Morgachev A.S., et al. // Sol. Phys., 2018, vol. 293, № 3, id 50.

7. Fleishman, G.D., Kontar, E.P. // Astrophys. J. Lett., 2010, 709, L127.

8. Zaitsev, V.V., Stepanov, A.V., Melnikov, V.F. // Astron. Lett., 2013, 39, 650.

9. Krucker, S., Giménez de Castro, C.G., Hudson, H.S. et al. // A\&A, Rev., 2013, 21, 58.

10. Morgachev A.S., Tsap Yu.T., Smirnova V.V., Motorina G.G. // Ge\&Ae, 2020, (in press).

11. Kaufmann P.; Trottet G.; Giménez de Castro, et al. // Sol. Phys, 2009, V. 255, Iss. 1, pp.131-142.

12. Trottet G., Raulin J.-P., Gimenez de Castro G., et al. // Sol. Phys., 2011, V. 273, Iss. 2, pp.339-361.

13. Morgachev A.S., Tsap Yu.T., Smirnova V.V., Motorina G.G. // Ge\&Ae, 2018, Vol. 58, No. 8, pp. 1113-1122, DOI: 10.1134/S001679321808011X.

14. Hanser, F.A. and F.B. Sellers, 1996, 2812, 344-352, doi: 10.1117/12.254082.

15. Hudson H.S., Simoes P.J.A., Fletcher L., et al. 2020, eprint arXiv:2007.05310.

16. Morgachev A.S., Tsap Yu.T., Smirnova V.V., Motorina G.G. // Ge\&Ae, 2019, Vol. 59, No. 8, pp. 1114-1120, DOI: 10.1134/S0016793219080140. 